\title{
Reversible Neurological Adverse Reaction to Apixaban
}

\author{
Eduardo Rodrigues Cernadas, Catarina Dionisio, Dália Estevão, Leopoldina Vicente \\ Centro Hospitalar Universitário Cova da Beira, Covilhã, Portugal
}

Doi: 10.12890/2021_001739 - European Journal of Case Reports in Internal Medicine - @ EFIM 2021

Received: $21 / 05 / 2020$

Accepted: $27 / 05 / 2020$

Published: 16/04/20201

How to cite this article: Rodrigues Cernadas E, Dionisio C, Estevão D, Vicente L. Reversible neurological adverse reaction to apixaban. EJCRIM 2021;8: doi:10.12890/2021_001739.

Conflicts of Interests: The Authors declare that there are no competing interests.

This article is licensed under a Commons Attribution Non-Commercial 4.0 License

\section{ABSTRACT}

Prescriptions for the novel oral anticoagulant factor Xa inhibitors (rivaroxaban, apixaban, edoxaban) have equalled or exceeded those for vitamin $\mathrm{K}$ antagonists in many clinical settings requiring chronic anticoagulation, and those of injectable heparins for deep vein thrombosis prophylaxis. The authors report the case of an 80-year-old woman followed by her cardiologist for permanent atrial fibrillation who was prescribed apixaban. Within a few days the patient developed neurological symptoms of imbalance and non-vertiginous dizziness, headache, confusion/disorientation and asthenia. Her symptoms began to resolve after the drug was stopped, with return to baseline function within $72 \mathrm{~h}$. The plasma concentration of apixaban was 4 times higher than the laboratory upper limit of normal. Symptoms did not recur when the patient was switched to rivaroxaban therapy.

\section{LEARNING POINTS}

- $\quad$ Prescribing of the novel oral anticoagulant factor Xa inhibitors is increasing.

- Reversible neurological effects may occur in some patients, for instance in those with advanced age or chronic renal failure.

\section{KEYWORDS}

Apixaban, neurological symptoms, adverse drug reaction

\section{INTRODUCTION}

New oral anticoagulants have gained in popularity over the traditional vitamin $\mathrm{K}$ antagonists. As they are relatively new to the market, the extent of adverse drug reactions continues to be characterized. The authors present the case of a patient treated with apixaban for atrial fibrillation stroke prophylaxis, who experienced complex neurological symptoms which resolved completely following drug discontinuation. Measurement of apixaban plasma concentration showed it was far above the upper limit of normal. We discuss the categorization of adverse drug events and explore possible mechanisms.

\section{CASE DESCRIPTION}

The authors report the case of an 80-year-old female patient followed by her private cardiologist for atrial fibrillation. Her medical history included dyslipidaemia, hypertension, depression, anxiety and diabetes mellitus (DM). She had no recent admission to the emergency department or general ward. She had only one son and was living alone in her own home. She did not consume alcohol or smoke. Her family history was significant for breast cancer, DM, hypertension and coronary heart disease. Her long-term oral medications only included 100 mg aspirin, $5 \mathrm{mg}$ diazepam and $125 \mu \mathrm{g}$ levotryroxine. She had no known allergic reactions to drugs or food. 
Her most recent laboratory studies including a complete metabolic profile, complete blood count, serum ferritin, fasting lipids, and thyroid stimulating hormone were normal. The patient had attended a private cardiology consultation for palpitations, having been diagnosed with atrial fibrillation, and was started on anticoagulation with apixaban $5 \mathrm{mg}$ twice daily (CHA2DS2VASc score 2). Shortly after the first dose, the patient began experiencing loss of balance, a sensation of weakness and headaches that worsened over the following days. By day 6 the patient was experiencing severe myalgia together with inability of the lower limbs to stand up in the morning after a night's sleep. The patient attended her general physician who suggested discontinuing apixaban and referred her for consultation to the internal medicine department at the hospital. The patient's complaints improved greatly after a few days, so before hospital contact, she decided to re-start apixaban at the same dose, but a few days later experienced the exact same symptoms as before.

Following the internal medicine consultation, the plasma concentration of apixaban was measured in the laboratory and showed values that were four times higher than the normal upper limit. The anticoagulant was changed to rivaroxaban 15 mg twice daily. After 4 months of treatment, the patient has experienced no similar or other adverse symptoms.

\section{DISCUSSION}

New (novel)/direct oral anticoagulants (NOACs, DOACs) that act through reversible factor Xa inhibition (rivaroxaban or apixaban) are now commonly used in lieu of warfarin in several clinical settings. In comparison to traditional warfarin, this group of drugs appeared, therapeutically at least, non-inferior for non-valvular atrial fibrillation-related stroke prophylaxis, deep vein thrombosis (DVT) prophylaxis, and DVT/pulmonary embolism treatment and are as safe or even safer regarding major bleeding ${ }^{[1]}$.

These drugs do not require regular blood monitoring and, apart from the additive effects of anticoagulant agents ${ }^{[1,2]}$, have far fewer drugdrug interactions ${ }^{[1]}$. They are easily administered once or twice daily and no interactions with food have been reported. Some patients with renal dysfunction, extreme age or low bodyweight may require dosing adjustment and only patients with severe liver disease are advised against the use of $\mathrm{NOACs}^{[3]}$.

Apixaban's pharmacokinetic profile has been described and includes a peak single dose concentration at about $3 \mathrm{~h}$, and an apparent $\beta$ half-life of about $12 \mathrm{~h}$ after multiple dosing ${ }^{[4]}$. The pharmacodynamic effects within a 10 -fold dose range (2.5-25 mg twice daily) are concentrationrelated with low to moderate variability. Of note, AUCtau between day 1 and day 7 administration of a 5 mg twice daily dose increased by $75 \%$. The drug is approximately $50 \%$ bioavailable after recommended dosing. About $25 \%$ of an oral dose is excreted as metabolites. O-demethylation and hydroxylation is primarily performed by CYP 3A4, although other cytochrome P450 enzymes (1A2, 2C8, 2C9, 2C19, 2J2) contribute to its metabolism.

The majority of adverse drug events reported for apixaban were related to one or more manifestations of heightened bleeding risk ${ }^{[3,4]}$. Some reports have also documented rare idiosyncratic drug-induced liver injury, which is usually, but not always, reversible with drug discontinuation ${ }^{[5]}$.

There are no literature reports of neurological adverse events due to apixaban, although an association with haemorrhage or haematoma formation has been noted. A major drug reference database ${ }^{[3]}$ lists pre-syncope (considered a cardiovascular effect) in $<1 \%$ of patients with non-valvular atrial fibrillation taking apixaban based on two large randomized trials/registries (ARISTOTLE trial, AVERROES Trial), but further details are not available to assess whether these patients might in fact have had complication syndromes similar to that of our patient. Other sources ${ }^{[5]}$ describe dizziness, confusion and decreased alertness, or headaches.

Several aspects of this case strongly suggest apixaban as the causative agent as: (a) symptoms were not present prior to drug administration; (b) symptoms began shortly after initial administration of this newly prescribed agent; (c) symptoms progressively worsened with continued drug consumption; (d) symptoms resolved rapidly with drug discontinuation; (e) symptoms recurred with (patient-initiated) re-challenge; and (f) the second episode of symptoms again resolved with drug discontinuation. CNS imaging was not considered cost-beneficial for this patient at the time of presentation, as the history of reproducible and totally resolving clinical symptoms made vascular or haemorrhagic events extremely unlikely ${ }^{[1]}$.

Adverse drug reactions are generally classified into six categories [3]: (a) dose-dependent, augmentations of known pharmacological activities; (b) non-dose-dependent, unpredictable events not related to the known pharmacology of the drug (includes immunological/ hypersensitivity and idiosyncratic reactions; implies host susceptibility through genetics or other mechanisms); (c) chronic (dose- and timerelated) reactions; (d) delayed (time-related) reactions; (e) withdrawal phenomena with drug discontinuation; and (f) unexpected failure of therapy.

The exact mechanism of our patient's adverse reaction is unclear, as signs and symptoms appear to cross standard categories. However, as these more convenient and likely superior, safer drugs become the standard of care for many large patient populations requiring chronic anticoagulation, post-marketing peer-reviewed reports of idiopathic adverse drug reactions to NOACs, such as that experienced by our patient, are critical. 


\section{REFERENCES}

1. Granger CB, Alexander JH, McMurray JJ, Lopes RD, Hylek EM, Hanna M, et al. Apixaban versus warfarin in patients with atrial fibrillation. N Engl J Med 2011;365(11):981-992.

2. Nishimura RA, Otto CM, Bonow RO, Carabello BA, Erwin JP 3rd, Guyton RA, et al. 2014 AHA/ACC guideline for the management of patients with valvular heart disease: a report of the American College of Cardiology/American Heart Association Task Force on practice guidelines. Circulation 2014;129(23):e521-e643.

3. Agnelli G, Buller HR, Cohen A, Curto M, Gallus AS, Johnson M, et al. Oral apixaban for the treatment of acute venous thromboembolism. N Engl J Med 2013;369(9):799-808.

4. Giugliano RP, Ruff CT, Braunwald E, Murphy SA, Wiviott SD, Halperin JL, et al. Edoxaban versus warfarin in patients with atrial fibrillation. N Engl J Med 2013;369(22):20932104.

5. Frost C, Shenker A, Gandhi MD, Pursley J, Barrett YC, Wang J, et al. Evaluation of the effect of naproxen on the pharmacokinetics and pharmacodynamics of apixaban. Br J Clin Pharmacol 2014;78(4):877-885. 